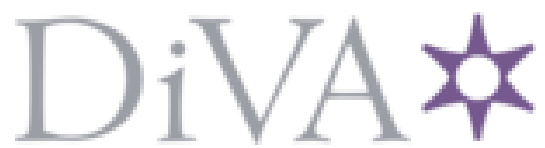

http://www.diva-portal.org

\title{
Postprint
}

This is the accepted version of a paper presented at 2018 IEEE 8th International ModelDriven Requirements Engineering Workshop (MoDRE,Banff, Alberta, Canada.

Citation for the original published paper:

wilson, M., Wnuk, K. (2018)

Towards Multi-context Goal Modeling and Analysis with the Help of Intents

In: 2018 IEEE 8th International Model-Driven Requirements Engineering Workshop

(MoDRE) IEEE Computer Society Digital Library

https://doi.org/10.1109/MoDRE.2018.00015

N.B. When citing this work, cite the original published paper.

Permanent link to this version:

http://urn.kb.se/resolve?urn=urn:nbn:se:bth-17337 


\section{Towards Multi-context Goal Modeling and Analysis with the Help of Intents}

\author{
Magnus Wilson \\ Ericsson, Karskrona, Sweden \\ Magnus.Wilson@ericsson.com
}

\author{
Krzysztof Wnuk \\ Department of Software Engineering \\ Blekinge Institute of Technology, Karlskrona, Sweden \\ Krzysztof.Wnuk@bth.se
}

\begin{abstract}
This vision paper discusses the need for enriching goal modeling with intents and context frame to provide much richer contextual information over goals and realization strategies. Based on the extensive experience of business modeling at Ericsson and a review of current goal modeling approaches, we suggest possible research directions towards enriching goal modeling.
\end{abstract}

\section{INTRODUCTION}

Goal modeling is an important part of Requirements Engineering, and several researchers focused on developing goal-modeling notations and methods [8]. I* [24] and KAOS [23] are the two most widely recognized goal modeling frameworks. Goal modeling support understanding why and support early-phases of requirements engineering, reason about non-functional aspects [5], modeling strategic relationships and social aspects [25] or dependencies between actors and their goals. However, goals do not capture the rich contextual information, the knowledge of the actors who specify them and the actors who receive and interpret them.

Another limitation is the limited support to capture the temporal nature of goals and their changeability over time. Although i* Strategic Rationale (SR) models can be used to show the internal intentional structures of actors, significant changes over time to the goals or actors usually result in creating new goal models. Despite some promising work on combining goal modeling (with $\left.i^{*}\right)$ and decision modeling [19], documenting rich contextual information, role and knowledge of the actors remains an area that is greatly unexplored.

This paper builds on theories (1) for how interaction between two parties create knowledge and understanding [20], [15], and (2) establishing the foundation for value creation through the intent via promises and agreements [1]. We present the result of extensive analysis of goal modeling principles and approaches performed by Ericsson $\mathrm{AB}$ and highlight the shortcoming and additional aspects that should be considered to increase the probability of industrial application of goal modeling.

We postulate that goal modeling should be enriched by intents (which are the state of mind with which an act is done ${ }^{1}$ ) expressed by context frames (as the total domain information for the specific domain an actor has obtained) [22]. Intents and context frames can provide a much richer and much-requested view of goals and realization strategies.

\section{Related Work about Goal Modeling AND CONVERSATION THEORY}

Understanding (business) goals and the associated intended value creation is an essential part of goal modeling. The business goals are derived from the business owner's intentions, which may vary over time. The value creation process aggregates the result of many interactions between actors, each based on complex relationships between the actor's needs, wants, knowledge, skills, and the contextual situation (role plus environment) [26], [10].

In this work, intent is defined as a subject or type of possible behavior, i.e. something that can be interpreted to have significance[1]. Any actor can have intents. We define an intent-driven system as a compositional system of human actors and machine actors. The machine actors are software agents where the agents declare, negotiate, and assess intents made by agents. The intents can be declared, negotiated, or assessed, on-behalf of an agent's stakeholder or an agent's self-interest. Intent-driven systems capture stakeholders' intents in the form of business requirements or capabilities and transform these intents into a form that enables computer processing of them [22]. This view is similar to the Belief-Desire-Intention (BDI) where human and computer agents are capable or rational behavior [21]. BDI recognizes the dynamics of contextual changes and different ways that agents and systems can respond to these changes. BDI also recognizes that a single agent has no possibility to capture all contextual changes, supporting our suggestions to introducing context frames and unified contextual information representation that can be shared between the agents.

Business models and business modeling are used to develop a greater understanding for the business

\footnotetext{
${ }^{1}$ By Merriam-Webster, https://www.merriamwebster.com/dictionary/intent
} 
and its' environment (context of the interactions). Business models capture the business goals, as connected to customers and partners, but also the underlying business logic, and how resources are configured to generate profit through transactions and interactions. The business model literature introduce three essential views for analyzing business goals: top-down [7], bottom-up [26], and inside-out [18], [17].

\section{A. Results of the analysis done at Ericsson}

By using Pask's conversation theory as a model for learning [20] and Nonaka et al. theory on dynamic knowledge creation [15] applicable to actors in a group, we can further elaborate on the details of intent and the temporal effects for an actor in an interaction. The two specific scenarios benefiting from this type of analysis apply to goal modeling.

- Scenario 1 (Define) deals with the interpretation and (mis-)understanding of the business goal between the two actors involved in goal modeling

- Scenario 2 (Execute) deals with the contents of the business analysis (value, products, stakeholders, business processes) and the definition of the business goal itself.

This work focuses primarily on Scenario 1, but also tackles Scenario 2, as the realization of any goal is done through a series of interactions in transactions. For efficient goal modeling, we postulate the importance of separating roles, contexts, and temporal effects in the models, and to be able to generate role-dependent views out of shared knowledge and information with the aid of efficient information management tools[22]. Our work is related to agent oriented software systems suggested by the Tropos methodology [3], [4], but highlight the importance of documenting and exchanging contextual information between the agents and/or actors .

Höflinger's conceptual framework of the Business model's antecedents and consequences see Figure 1, combined with the dynamics of interactions and learning provides us with a theoretical foundation to understand the interactions that take place to fulfill individual business goals (scenario 2). Combined with the activity system view by Zott et al. Figure 2 serves to explain the temporal aspects of goal evolution, derived from an individual actor's intent, knowledge, skills, and learning.

Each action performed on any of Höflinger's sub-component results in reactions (and changes) in other sub-components that can cascade and can cause potential misalignments of the business model (H3) and its' consequences (H4). The arrows in Figure 1 depict such continuous, multirelationship influence as a process of change, which in turn is the consequence of a multitude of in-

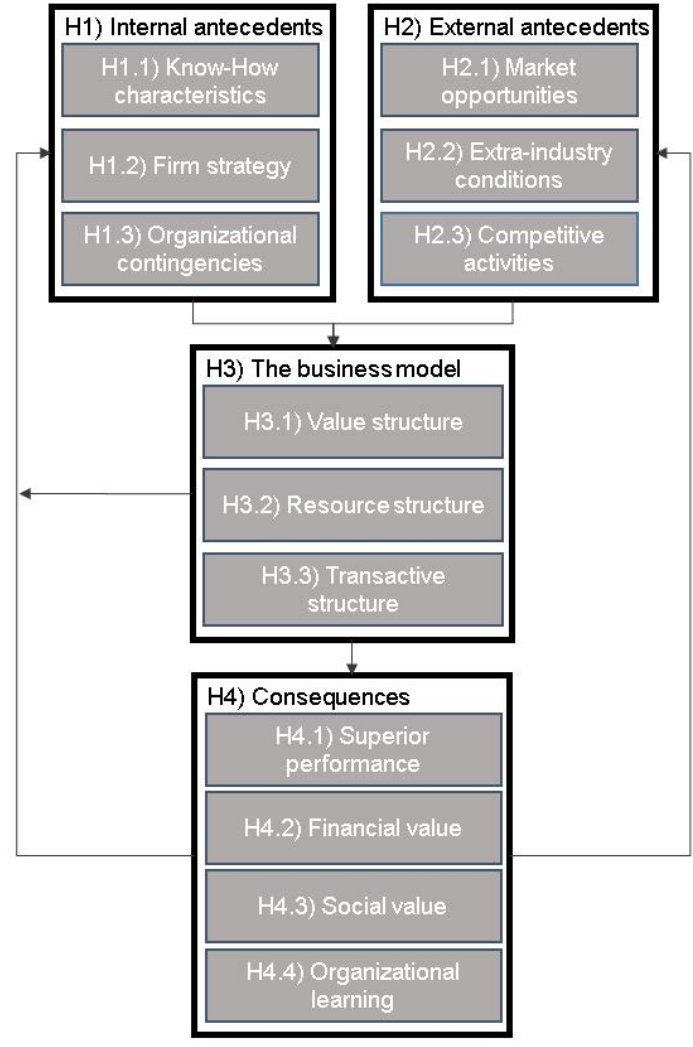

Figure 1. Goal modeling as a linear flow based on Höflinger's conceptual business model framework

teractions (IV) on different abstraction levels in combination with organizational learning (LV). Examples of abstraction levels are company level (value), organizational level (function/capability), and individual level (task/ability to execute) which are also identified by Mason \& Mouzas where they also discuss how flexibility is developed to re-enable alignment (in and between layers)[12].

These three abstraction levels also map to the top-down, inside-out, and bottom-up approach of business models as per our references. Business modeling can, therefore, be seen as a highly iterative and recursive process of experimentation, knowledge creation, and learning, toggling between the critical states of dialogue and practice [15] or as conversation theory explains how learning is created, through repeated cycles of explanation and demonstrated understanding [20].

\section{B. Interactions, transactions and business goals}

To explore how (and when) these transaction and interactions relate to the business goals and how different actors interpret, react, and (as an organization) learn from the outcome of the interaction, we add conceptualizations for Interaction (IV) and Learning (LV), see Figure 2.

The Interaction view (IV) in Figure 2 illustrates interactions between two actors and helps to explore the complex relationships and causalities 


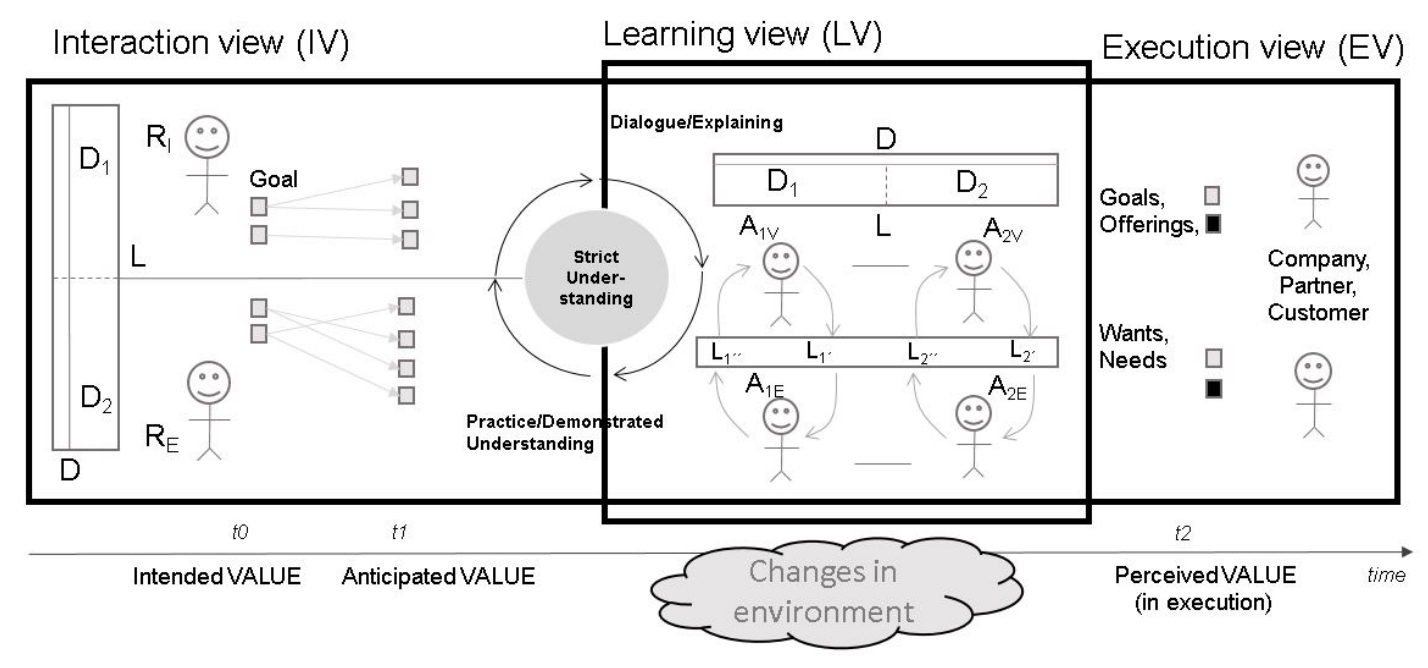

Figure 2. Shaping business goals via the Interaction view and Learning view, a conceptualization of two actors in an iterative interaction, applicable for any abstraction level

between (sub)-components (H1-H4), see Figure 1. An internal actor (RI) interacts with an external actor $(\mathrm{RE})$ using an agreed language $(\mathrm{L})$ related to the domain $\mathrm{D}(=\mathrm{D} 1+\mathrm{D} 2)$. Domain $\mathrm{D} 1$ is defined by the boundaries and constraints stemming from internal antecedents (H1) and the business model (H3) with the relation to RI. Domain D2 is derived from external antecedents $(\mathrm{H} 2)$ and the business model $(\mathrm{H} 3)$ in relation to RE. Both D1 and D2 are also part of a larger domain which shares many similarities with the " $B a$ " concept in the Knowledge creation process [14].

The internal and external actors in the interaction view (IV) may have different intents and slightly different interpretation of the shared domain $\mathrm{D}$, in which the interaction takes place. RI and RE are described by the resource structure (H3.2). If RE is a customer, additional descriptions are found in market opportunities (H2.1) and the value structure (H3.1), see Figure 1.

The context of the interaction is described by the transactive structure $(\mathrm{H} 3.3)$ in relation to resources and skills described in the resource structure (H3.2). The result of an interaction is therefore dependent, not only on the domain (Ba), but also connected to the dynamics between individual actors, i.e., need's fulfillment, value creation (guided by understanding), and learning. Domain knowledge and cross-functional communication plays the key role is these interactions [6].

The language $\mathrm{L}$ in Figure 2 can also be seen as a representation of the offer and contractual agreements derived from the value structure (H3.1). A goal for the interaction is to satisfy the needs of RE thereby creating a perceived value, optimally matching an offer (one or several anticipated values) as designed in the value structure (H3.1). By using an optimal configuration of value and control parameters (detailed by H1.1, H1.3, H3.1, H3.2, and $\mathrm{H} 3.3$ ) the intended consequences (H4) are also fulfilled for the company (represented by RI).

The consequences (H4) are divided into four key aspects where superior performance (H4.1) and financial value $(\mathrm{H} 4.2)$ are the obvious intended consequences of a business model. Höflinger differentiates between financial value (H4.2) and social value (H4.3) while our previous work on Software Value map [9] explores additional essential value aspects and for example, translates organizational learning (H4.4) into explicit innovation and intellectual capital values. Organizational learning (H4.4) can be seen as a feedback loop and how an organization develops knowledge to maintain superior performance and to create/rejuvenate innovative business models for the longer term via systematic business model innovation.

Learning (LV) in Figure 2 is added to explore the organizational learning and the dynamics of why and when there might arise a misalignment between reality and the intended consequences, as shaped in any interaction by original intents, skills, new understandings, and learning.

Learning (LV) in Figure 2 illustrates how knowledge is created in a conversation as a consequence of reaching strict understanding between two actors $\mathrm{A} 1 \mathrm{~V}$ and $\mathrm{A} 2 \mathrm{~V}$, who has agreed in a contract to follow the rules of a language $\mathrm{L}$ valid in the domain D according to Promise Theory [1]. Their respective understanding of $\mathrm{D}$ does not need to be the same, hence slightly different domains (D1, D2) in which a translation, interpretation, and exemplification to demonstrate understanding takes place (which may involve more actors/machines in forward and backward feedback loops, as illustrated 
by the languages L1', L1', L2', L2" and A1E, A2E). This model highlights the importance of requirements negotiation and communication and roles like information brokers may be essential here [11].

By reaching an understanding, knowledge is created and represented by topics becoming commonly known (and added to D). Actor A1V continues to explain until A2V illustrates understanding by exemplification and strict understanding is reached. This is a 2-phase process (Define and Execute) that involves toggling between definitions and concretization by exemplification. Applying such mechanism on the Interaction (IV), RI can be abstracted to a chain of actors, and if strict understanding is not reached, potential misalignment is introduced and will cascade with every interaction.

For scenario 1, an example of actors could be Product Manager (RI) trying to agree and align with the design suggested by the chief architect (RE) and discussing what goals to develop and how they impact the current business and business processes. For scenario 2, we simply replace the actors towards a company (RI) and a customer (RE), and the same mechanism applies on a different abstraction level, but in a much more complex environment due to the size and complexity of the whole value creation and value capture.

\section{Problem statement}

In today's digital business transformation [13], [2], we argue that the feedback loops must be supported by efficient role-based information management tools that can be efficiently and transparently used by all actors collaborating in the value capture and value creation activities. Given today's methods in RE, including goal modeling, the modeling methods and tools are not fast enough to allow all actors to collaborate efficiently to realize the business goals. As a consequence, Ericsson is actively looking into new ways of modeling (and information management tools) to maintain the scale and speed of change, with a predictable level of quality, as one strategy to manage the digital business transformation ${ }^{2}$.

\section{ENHANCING GOALS WITH INTENTS}

We believe goal modeling would benefit from the richer notion of changing business intents [22] by understanding how changing business intents evolve through interactions, see Figure 3. By looking how vision, mission, strategies, goals, tactics, and assessments relate to policies and rules (encoded knowledge) [16] and combining this with the Business Model Canvas [17], we define a

\footnotetext{
${ }^{2}$ Seen from an industry view, please watch this talk by Simon Wardley as an anecdotal introduction to the challenges of translating strategies into successful businesses, https://youtu.be/xlNYYy8pzB4
}

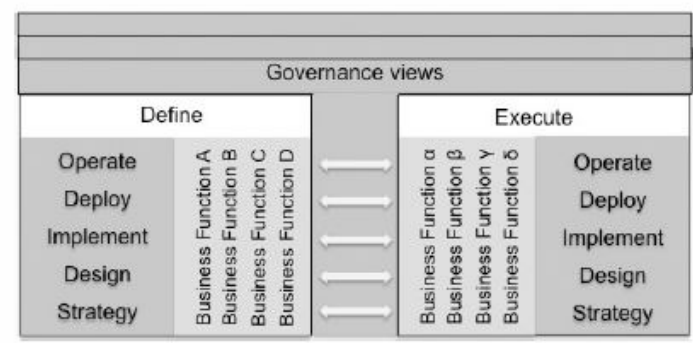

Figure 3. Deriving business goals from changing business intents

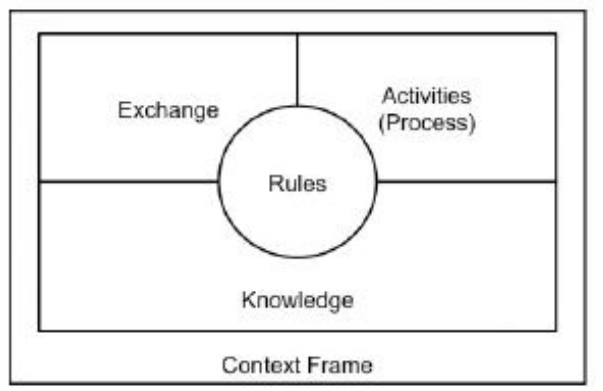

Figure 4. Metamodel for a context frame

conceptual model for what actor information is important to capture and share between other actors in different domains.

To achieve scalable and an abstraction-less information representation, we also define the context frame as "as the total domain information for the specific domain an actor has obtained" with the metamodel in Figure 4 [22]. The compositional context frame sits in the middle of the changing business intent's lifecycle, and via the concept of Governance views, all necessary information can be accessed and shared with all stakeholders in their interactions (on any abstraction level), see Figure 3. We also speculate that Digital Business modeling ${ }^{3}$ will become a fusion of existing RE, Software Product lines, and business modeling, in the same way as Digital strategy has become a fusion of business and IT strategy [2] as result of the new digital business where software become a vital part of products, business processes, and other tools for any company.

\section{IMPLICATIONS FOR RESEARCH AND PRACTICE}

One of the main implications from our work is the lack of fast feedback loops between the definition phase and the execution phase. We have also found no information representation that will

\footnotetext{
${ }^{3}$ Seen from an industry view, please read this SAP white paper as a brief introduction to Digital business modeling and the challenges for efficient and effective information management, https://tinyurl.com/y $77 \mathrm{~kg} 38 \mathrm{~m}$
} 
allow for contextual negotiation between actors to accommodate an alignment and feedback of anticipated (designed) and perceived value (via a contract). We believe such a mechanism to be crucial for enabling an experimental, collaborative business environment and ecosystem.

We believe that will soon see an explosion in 'smart agents', where human actors will be supported by software agents acting on their behalf, observing, interpreting, negotiating, deciding and fulfilling their intents based on available information and continuous learning. Of course, machinelearning, block-chains, and other technologies will be an integral part of such intent-driven systems, bringing challenges within transparency, privacy, security, legal accountability.

We believe that intent-driven systems based on context frames will create a new architectural style based on near real-time flexible governance views. Together with a new set of interfaces, software agents should be able to help humans manage changing business intents. For example, software agents can help part of our business processes become automated while adapted to understand and negotiate intents into promises and contracts, which can be acted upon. Software agents can also be built into the business, enterprise, and product software architectures, as the time between changing the business model until the new business model is operationalized will continue to decrease, while the required information that needs to be kept consistent is increasing as well as becoming decentralized.

\section{REFERENCES}

[1] Jan A. Bergstra and Mark Burgess. Promise Theory: Principles and Applications.

[2] Anandhi Bharadwaj, Omar A. El Sawey, Paul A. Pavlou, N. Venkatraman, Omar a. El Sawy, Paul A. Pavlou, and N. Venkatraman. Digital Business Strategy: Toward a Next Generation of Insights. MIS Quarterly, 37(2):471-482, 2013.

[3] Paolo Bresciani, Anna Perini, Paolo Giorgini, Fausto Giunchiglia, and John Mylopoulos. Tropos: An agentoriented software development methodology. Autonomous Agents and Multi-Agent Systems, 8(3):203-236, May 2004.

[4] Jaelson Castro, Manuel Kolp, and John Mylopoulos. Towards requirements-driven information systems engineering: the tropos project. Information Systems, 27(6):365 389, 2002.

[5] Lawrence Chung and Julio Cesar Sampaio do Prado Leite. On Non-Functional Requirements in Software Engineering, pages 363-379. Springer Berlin Heidelberg, Berlin, Heidelberg, 2009.

[6] D. Damian, R. Helms, I. Kwan, S. Marczak, and B. Koelewijn. The role of domain knowledge and crossfunctional communication in socio-technical coordination. In 2013 35th International Conference on Software Engineering (ICSE), pages 442-451, May 2013.

[7] Nicolas F. Höflinger. The business model concept and its antecedents and consequences - towards a common understanding. Academy of Management Proceedings: Organization Development \& Change, 2014:1, 2014.
8] Evangelia Kavakli and Pericles Loucopoulos. Goal modeling in requirements engineering: Analysis and critique of current methods. In Information modeling methods and methodologies: Advanced topics in database research, pages 102-124. IGI Global, 2005

[9] Mahvish Khurum, Tony Gorschek, and Magnus Wilson. The software value map - An exhaustive collection of value aspects for the development of software intensive products. Journal of software: Evolution and Process, 25(7):711$741,2013$.

[10] David P. Lepak, Ken G. Smith, and M. Susan Taylor. Introduction to special topic forum value creation and value capture: A multilevel perspective. Academy of Management Review, 32:180-194, 2007.

[11] S. Marczak, D. Damian, U. Stege, and A. Schröter. Information brokers in requirement-dependency social networks. In 2008 16th IEEE International Requirements Engineering Conference, pages 53-62, Sept 2008.

[12] Katy Mason and Stefanos Mouzas. Flexible business models. European Journal of Marketing, 46(10):13401367, 2012.

[13] Christian Matt, Thomas Hess, and Alexander Benlian. Digital transformation strategies. Business and Information Systems Engineering, 57:339-343, 2015.

[14] Ikujiro Nonaka and Noboru Konno. The concept of "ba": Building a foundation for knowledge creation. California Management Review, 40(3):40-54, 1998

[15] Ikujiro Nonaka, Ryoko Toyama, and Noboru Konno. SECI, Ba and Leadership: a Unified Model of Dynamic Knowledge Creation. Long Range Planning, 33(1):5-34, 2000.

[16] Omg.org. About the Business Motivation Model Specification Version 1.3. http://www.omg.org/spec/BMM/, 2015.

[17] Alexander Osterwalder and Yves Pigneur. Business model generation: A handbook for visionaries, game changers, and challengers.

[18] Alexander Osterwalder and Yves Pigneur. Designing business models and similar strategic objects : The contribution of is. Journal of the Association of Information Systems, 14:237-244, 2013.

[19] Vik Pant and Eric S. K. Yu. Understanding strategic moves and reciprocity on software ecosystems: A strategic modeling approach. In Proceedings of the 9th International Workshop on Software Ecosystems, Espoo, Finland, November 29, 2017., pages 28-42, 2017.

[20] Gordon Pask, To Elisabeth, and Amsterdam-oxford-new York. Conversation Theory - Applications in Education and Epistemology. Elsevier Inc., Amsterdam and New York, 1976.

[21] Anand S Rao, Michael P Georgeff, et al. Bdi agents: from theory to practice. In ICMAS, volume 95, pages 312-319, 1995.

[22] Johan Silvander, Magnus Wilson, Krzysztof Wnuk, and Mikael Svahnberg. Supporting continuous changes to business intents. International Journal of Software Engineering and Knowledge Engineering, 27:1167-1198, 2017.

[23] A. van Lamsweerde. Goal-oriented requirements engineering: a guided tour. In Proceedings Fifth IEEE International Symposium on Requirements Engineering, pages 249-262, 2001.

[24] E. S. K. Yu. Towards modelling and reasoning support for early-phase requirements engineering. In Requirements Engineering, 1997., Proceedings of the Third IEEE International Symposium on, pages 226-235, Jan 1997.

[25] Eric Yu, Paolo Giorgini, Neil Maiden, and John Mylopoulos. Social Modeling for Requirements Engineering. The MIT Press, 2011.

[26] Christoph Zott and Raphael Amit. Business Model Design: An activity system perspective. Long Range Planning, 43(2-3):216-226, 2010. 